\title{
Preoperative axillary lymph node staging by ultrasound-guided cytology using a four-level sonographic score
}

\author{
Caroline De Coninck*, Jean-Christophe Noël, Rachel Boutemy and Philippe Simon
}

\begin{abstract}
Background: The staging of axillary lymph nodes is critical to the management and prognosis of breast cancer, the most frequent cancer in females. Neoadjuvant therapy and lymph node dissection are recommended when malignant cells invade the lymph nodes. Therefore the pre-operative examination of these lymph nodes is crucial to treatment.

Methods: In this study, we examined the effectiveness of cytology through ultrasound-guided fine needle aspiration (USG-FNA) and ultrasound (US) imaging using an established classification system in correctly identifying lymph node status compared to the final histological results after surgery.

Results: Cytology by USG-FNA and US classification were found to be promising methods of axillary lymph node staging.

Conclusions: US and CB offer minimally invasive techniques to pre-operatively examine these lymph nodes in patients with primary breast cancer.
\end{abstract}

Keywords: Axillary lymph nodes, Breast cancer, Ultrasound, Cytology, Metastasis

\section{Background}

Breast cancer is the most frequent female cancer [1] and the second leading cause of female cancer death in Belgium [2]. Therefore, breast cancer management is critically important. When an axillary lymph node is invaded, neoadjuvant chemotherapy and axillary lymph node dissection are indicated $[3,4]$. In terms of survival, it is now widely accepted that there are advantages of neoadjuvant chemotherapy in patients with nodepositive breast cancer [3, 4]. Axillary lymph node status is an important factor in the prognosis and management of breast cancer. Several methods to detect positive axillary lymph nodes during the pre-operative diagnosis have been evaluated, including ultrasound-guided fine needle aspiration (USG-FNA) cytology, ultrasoundguided biopsy and, as an imaging method, axillary ultrasound [5]. If a positive lymph node is not found during the pre-operative evaluation, a less invasive sentinel node biopsy will be utilized instead of axillary lymph

\footnotetext{
*Correspondence: caroline.de.coninck@ulb.ac.be

Erasme Hospital, 808 route de Lennik, 1070 Anderlecht, Belgium
}

node dissection [6]. The goal of this study was to compare the results of axillary lymph node status by cell-block obtained through fine-needle aspiration [7] and by axillary ultrasound, according to a classification system derived from Stavros [8].

\section{Methods \\ Patient dataset}

The Hoppital Erasme ethics committee approved this study. All the results were analyzed retrospectively and anonymously.

This study included a series of 208 cell or cytoblocks (CB) of axillary or para-axillary (chest wall, subclavicular and intra-mammary) lymph nodes from 184 patients (141 patients with one, 18 patients with two, one patient with three, and one patient with four evaluated lymph nodes) with primary breast cancer from a university center collected between October 2008 and August 2012.

Fine-needle aspirations were performed on all patients by a radiologist using a 21-gauge, ultrasonographic (US)- 
guided needle prior to any operative procedure on the breast or axilla, regardless of whether the nodes appeared normal or abnormal.

\section{Lymph node classification}

Lymph nodes were classified into four categories according to criteria derived from Stavros' classification: normal "N", minimally suspect "+", mildly suspect " ++ " and highly suspect " +++ ". The details of these classifications are as follows:

- "+": lymph node with a maximum size of cortical thickness of $3 \mathrm{~mm}$ with regular capsular thickening

- "++": lymph node with irregular capsular thickening (with notches) or with regular capsular thickening and cortical thickness greater than $3 \mathrm{~mm}$ in size

- "+++": complete loss of lymph node structure; irregular cortex or absence of lymph node hilum.

Normal or "+" lymph nodes were considered nonsuspicious, and "++" and "+++" lymph nodes were considered suspicious (Fig. 1).

\section{Cytoblock and cytospin techniques}

The sample obtained by US-guided fine-needle aspiration (USG-FNA) was placed in Saccomanno fluid and sent to the laboratory where it was separated into two samples: one for the $\mathrm{CB}$ technique and one for the cytospin technique. Both samples were centrifuged at $1400 \mathrm{rpm}$ for $10 \mathrm{~min}$. The cytospin sample was placed in a Shandon EZ Cytofunnel ${ }^{\circ}$ with a few drops of concentration solution, then placed in the Shandon Cytospin 4 centrifuge for $10 \mathrm{~min}$ at $500 \mathrm{rpm}$. It was then placed on a microscope slide for cytologic analysis. For CB, 2-4 drops of Reagent 2 from the cytoblock kit were added to the pellet, and it was resuspended and incubated for one minute. Two to four drops of Reagent 1 from the kit were added, causing polymerization. After one minute, the resulting polymerized material was placed between two sponges in a standard cassette and routinely processed with other biopsies and paraffinembedded blocks. Four-micron sections were cut and stained with hematoxylin and eosin. Immunohistochemistry (IHC) was performed on each cytoblock using broad-spectrum cytokeratin primary antibodies (clone AE1-AE3, dilution: 1/100, Dakocytomation, Glostrup, Denmark).

The $\mathrm{CB}$ results were classified into one of three categories: "C1" inadequate, "C2" negative and "C5" malignant.

\section{Histological assessment}

From the 208 CBs, only 93 had final histological confirmation of axillary lymph nodes after axillary lymph node dissection or sentinel node biopsy. These were categorized as either negative, when all of the examined lymph nodes were negative for metastasis, or positive, when there was evidence of metastasis in one or more lymph nodes. The same histopathologist performed histological assessments. Of the 93 results, only 54 had no neoadjuvant chemotherapy and were thus interpretable (Fig. 2).

The results were analyzed according to the type of breast cancer, the size of the evaluated lymph nodes, type of surgery (sentinel node biopsy or axillary lymph node dissection), surgeon, radiologist, patient's age, patient's BMI, and the presence or absence of neoadjuvant chemotherapy.

\section{Results}

Ninety-three CBs from 80 patients who underwent surgery had histological confirmation of axillary lymph nodes after sentinel node biopsy or axillary lymph node dissection. The 80 patients were between 28 and 84 years old. Tumor size was between 4 and $85 \mathrm{~mm}$, and the axillary lymph node size was between 5 and $42 \mathrm{~mm}$. In 73 cases, an axillary lymph node dissection was performed, and in 20 cases a sentinel node biopsy was performed.

Because 39 patients received neoadjuvant chemotherapy, the histological results from their surgeries were not interpretable. In effect, only the 54 patients who did not receive neoadjuvant chemotherapy were analyzed. Among these, 15 had non-suspicious lymph nodes $(\mathrm{N}), 23$ were minimally suspect, nine were mildly suspect, and seven were highly suspect (Table 1). Five CBs were inadequate, 39 were negative, and 10 were positive (Table 2).

Of the 54 cases without neoadjuvant chemotherapy, 34 had a negative histological result and 20 had a positive histological result. Of the 34 negative results, 14 had an echo of N, 18 echo of + (also considered nonsuspicious) and only two had an echo of ++ . Of the 20 positive results, one had an echo $\mathrm{N}$, five had + echoes, seven had ++ echoes, and seven had +++ echoes.

Of the 54 cases without neoadjuvant chemotherapy, 34 had a negative histological result and 20 had a positive histological result. Of the 34 negative results, only 2 had non-contributory $\mathrm{CB}(\mathrm{C} 1), 32$ had negative $\mathrm{CB}(\mathrm{C} 2)$ and there were no positive $\mathrm{CB}(\mathrm{C} 5)$. Of the 20 positive results, 3 had non-contributory $\mathrm{CB}(\mathrm{C} 1), 7$ had negative CB (C2) and 10 had positive CB (C5).

The final histological result was negative for 34 cases and positive for 20 cases.

Of the 38 non-suspicious lymph nodes based on US ( $\mathrm{N}$ or " + ") in patients who did not have neoadjuvant therapy, lymph node metastasis was found in six cases $(15.8 \%)$, whereas there were no malignant cells in 32 cases $(84.2 \%)$ (Table 1$)$. 


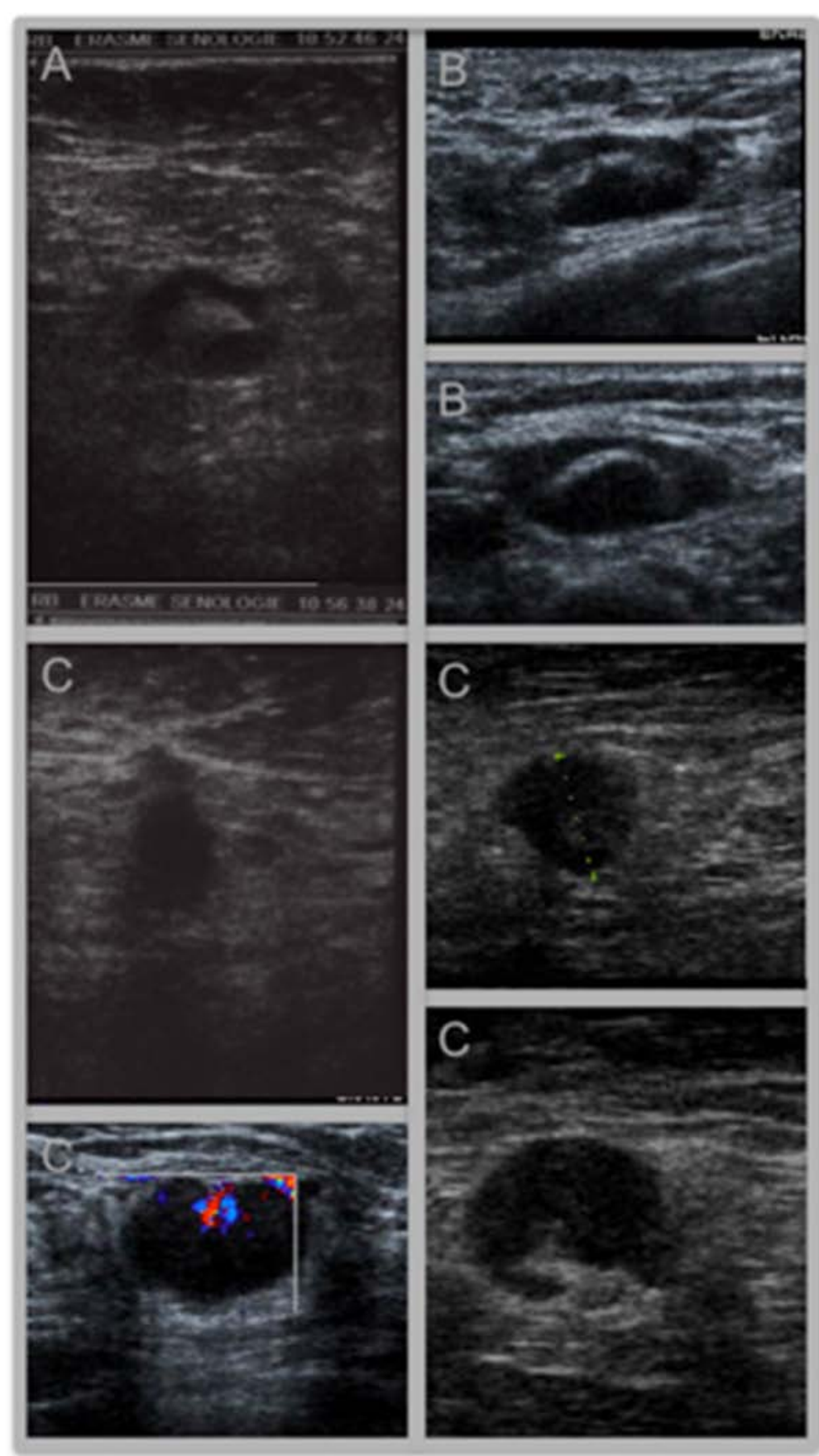

Fig. 1 Sonographic images of a minimally suspect axillary lymph node " + " (A), a mildly suspect axillary lymph node " ++ " (B) and highly suspect axillary lymph node " +++ " (C)

Of the 16 suspicious lymph nodes based on US ("++" or "+++") in patients who did not receive neoadjuvant therapy, lymph node metastasis was found in 14 cases $(87.5 \%)$, and no malignant cells were detected in two cases $(12.5 \%)$ (Table 1$)$.

All metastatic axillary lymph nodes identified by $\mathrm{CB}$ preparation in the absence of neoadjuvant chemotherapy
(C5) had positive final histological results (100 \%) (Table 2).

Of the 39 axillary lymph nodes in which metastasis was not found by $\mathrm{CB}$ preparation (C2), 32 had negative final histological results in the absence of neoadjuvant chemotherapy (82\%), and seven had positive final histological results (18\%) (Table 2). 


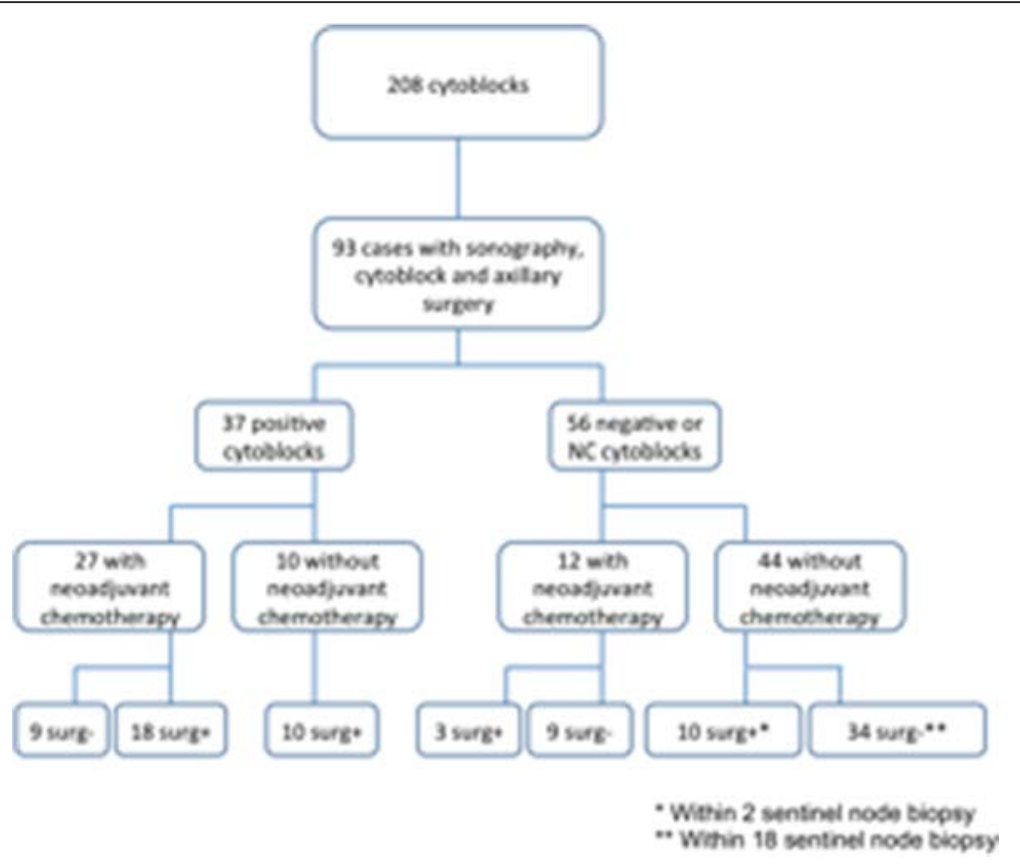

Fig. 2 Two hundred and eight cytoblocks (CB) were performed at Erasme Hospital between October 2008 and August 2012. Of these, 93 cases had sonography, cytoblock and axillary surgery. Of the CBs performed, 37 were positive and 56 were negative or non-contributory (NC). Of the 37 positive CB, 10 did not receive neoadjuvant therapy. Of the 56 negative or NC cytoblocks, 44 did not receive neoadjuvant chemotherapy

The results of the pre-operative lymph node staging by US and by $\mathrm{CB}$ preparation with the final histological result were compared using a $\chi^{2}$ statistical test. A statistically significant correlation was found between the results in the two cases (Table 2). The results obtained were statistically significant $\left(\chi^{2} 23.691(p=0.00000717)\right.$ for CB and $\chi^{2} 25.381$ ( $\left.p=0.00000046\right)$ for US.

Of the C5 lymph nodes (10 cases) or those found by US to be suspicious ( 9 "++" and 7 " +++ ") in the preoperative evaluations (26 cases), only two cases of " ++ " were ultimately negative in the absence of neoadjuvant therapy in the final histological results.

To determine whether some of the variables could explain a discordance in the results, or whether the rate of contribution changed depending on these variables, the impact of the variables on the results were analyzed using a $X^{2}$ statistical test. None of the variables were found to be statistically significant.

Table 1 Lymph nodes histological results according to pre-operative sonographic results

\begin{tabular}{lllll}
\hline \multicolumn{5}{c}{ Histological results from surgery } \\
\hline Echography & & Negative (\%) & Positive (\%) & Total \\
& Echo $N$ & $14(93)$ & $1(7)$ & $15(100)$ \\
& Echo + & $18(78)$ & $5(22)$ & $23(100)$ \\
& Echo ++ & $2(22)$ & $7(78)$ & $9(100)$ \\
Echo +++ & 0 & $7(100)$ & $7(100)$ \\
Total & $34(63)$ & $20(27)$ & $54(100)$ \\
\hline
\end{tabular}

In comparing the results of the $\mathrm{CB}$ preparation to those of the cytospins (57 cases), only three cases were discordant: three cases of inadequate $\mathrm{CB}$ had cytospin results of $\mathrm{C} 3, \mathrm{C} 4$ or $\mathrm{C} 5$.

A comparison of axillary lymph node status between $\mathrm{CB}$ preparation or US, and the final histological results of lymph nodes after surgery in the absence of neoadjuvant chemotherapy, indicated that false-negative results for metastasis were identified by $\mathrm{CB}$ in seven cases $(7 / 39,18 \%)$ compared to one " $\mathrm{N}$ " $(1 / 15,6.67 \%)$ and five "+++" (5/23, $21.7 \%)$ cases obtained by US alone $(6 / 38$, $15.8 \%)$. Moreover, the CB technique showed $0 \%$ falsepositive cases compared to $12.5 \%$ (two "++", 16 "++", and "+++") false-positive cases obtained by US alone. The results summarized in Table 3 show the sensitivities, specificities, positive predictive values (PPV) and negative predictive values (NPV) for US alone compared to $\mathrm{CB}$, excluding $\mathrm{CB}$ cases with inadequate results, and patients who received neoadjuvant chemotherapy.

Table 2 Lymph nodes histological results according to pre-operative cytoblock results

\begin{tabular}{lllll}
\hline \multicolumn{5}{c}{ Histological results from surgery } \\
\hline Cytoblock & \multicolumn{5}{l}{ Negative (\%) } & Positive (\%) & Total \\
& C 1 & $2(40)$ & $3(60)$ & $5(100)$ \\
C 2 & $32(82)$ & $7(18)$ & $39(100)$ \\
C 5 & 0 & $10(100)$ & $10(100)$ \\
& Total & $34(63)$ & $20(37)$ & $54(100)$ \\
\hline
\end{tabular}


Table 3 Statistical peformance

\begin{tabular}{lllll}
\hline & $\begin{array}{l}\text { Sensibility } \\
(\%)\end{array}$ & $\begin{array}{l}\text { Specificity } \\
(\%)\end{array}$ & $\begin{array}{l}\text { PPV } \\
(\%)\end{array}$ & $\begin{array}{l}\text { NPV } \\
(\%)\end{array}$ \\
\hline Echography* & 68.4 & 94.1 & 86.7 & 84 \\
Cytoblock & 58.8 & 100 & 100 & 82.1 \\
$\begin{array}{l}\text { Echography* }+ \\
\text { Cytoblock }\end{array}$ & 75.0 & 94.1 & 88.2 & 86.5 \\
${ }^{*}$ Echography N and + considered as non suspicious; echography ++ and +++ \\
considered as suspicious
\end{tabular}

Based on the values displayed in Tables 1 and 2, we calculated the sensitivity, specificity, positive predictive value (PPV) and negative predictive value (NPV) of echography* and cytoblock both alone and together. "Echoes of $\mathrm{N}$ and + were considered non-suspicious; echoes of ++ and +++ considered suspicious.

For the "++" and "+++" lymph nodes classified as suspicious in the absence of neoadjuvant therapy, the sensitivity of $\mathrm{CB}$ in the 54 cases was $58.8 \%$ compared to $68.4 \%$ for US. The specificity (100\% versus $94.1 \%$ ), PPV (100\% versus $86.7 \%)$ and NPV (82.1 \% versus $84 \%)$ values were high for both diagnostic methods (Table 3).

In cases where only the "+++" lymph nodes were considered suspicious, the sensitivity of $\mathrm{CB}$ in the 54 cases was $58.8 \%$ compared to $85.7 \%$ for US. Specificity was $100 \%$ versus $100 \%$, PPV was $100 \%$ versus $100 \%$, and NPV was $82.1 \%$ versus $93.3 \%$ (Table 3 ).

For non-suspicious lymph nodes with $\mathrm{N}$ or "+" results from US and $\mathrm{C} 2$ (a combination of the $\mathrm{CB}$ and US techniques) without neoadjuvant therapy, the sensitivity was $75 \%$, specificity was $94.1 \%$, PPV was $88.2 \%$ and NPV was $86 \%$ (Table 3).

These results support the hypothesis that pre-operative CB from USG-FNA biopsy of axillary lymph nodes, and axillary US using a modified version of Stavros' classification, are two promising methods of pre-operative axillary staging, with axillary US being the preferred means of staging.

\section{Discussion}

This study is based on a series of CBs from USG-FNA and sonography of axillary lymph nodes in patients with primary invasive breast cancer. Among non-invasive approaches, US and USG-FNA cytology have been reported to have high accuracy for staging axillary lymph nodes. Published estimates of USG-FNA cytology show a sensitivity varying from 25 to $87 \%$, a specificity ranging from 14 to $100 \%$, an NPV between 54 and $78 \%$ and a PPV ranging from 37 to $100 \%$ [6, 7, 9-13]. The results obtained in our study are similar to and even exceed these published findings. The only study to have previously described results from the CB technique, Engohan et al., has results very similar to those described here, with a better sensitivity (73 \% versus $58 \%$ ), a lower NPV (78 \% versus $82 \%$ ) and same specificity and PPV (100\%). Analysis of our results showed that for patients with positive nodes by CB (C5) or positive nodes by US ("+++"), the probability of having axillary nodal metastasis upon surgery in the absence of neoadjuvant therapy is $100 \%$ (PPV). Similarly, for patients with negative nodes by the CB technique and by US ( $\mathrm{N}$ or “+”), the probability of having no axillary nodal metastasis upon surgery is $86.5 \%$ (NPV) in the absence of neoadjuvant chemotherapy.

We found seven false-negative cases by the CB technique and by US, including one that had an inadequate $\mathrm{CB}$ result. Another false-negative lymph node was evaluated simultaneously with an ipsilateral lymph node that was positive at pre-operative staging; surgery showed two positive lymph nodes out of 12 analyzed. In effect, the second node was confirmed as positive without insurance, because the pathologist could not prove which one was the one diagnosed before surgery. We also found two false-positive cases by US when the CB result was negative, and the size of the pre-operative lymph node, as determined by US, was not the same as that measured by the pathologist after surgery.

This analysis reveals some limitations to our study. First, it is possible that the lymph node analyzed by the pathologist after surgery (sentinel node biopsy or axillary lymph node dissection) was not the same one as that evaluated by US and USG-FNA during preoperative staging.

Additionally, there were 39 cases of lymph nodes from patients who had received neodajuvant chemotherapy. For those cases, it is not possible to compare the preoperative and final histological results.

\section{Conclusion}

Axillary lymph node status is an important component of staging and treatment planning in breast cancer. Our study confirms that the use of a combination of US and $\mathrm{CB}$ may improve the evaluation of axillary lymph nodes in patients with primary breast cancer. These techniques are simple, inexpensive, minimally invasive, and allow for immunostaining. They also permit the referral of patients with primary breast cancer to neoadjuvant chemotherapy if the CB result is C5 or if the US result is "+++". In the same way, for patients with a CB result of $\mathrm{C} 2$ and US result $\mathrm{N}$ or " + ", these techniques may preclude the use of neoadjuvant chemotherapy. Given the limited number of cases in our study, additional, new studies will be necessary.

\section{Abbreviations}

CB: cytoblock; IHC: immunohistochemistry; NPV: negative predictive value; PPV: positive predictive value; US: ultrasound; USG-FNA: ultrasound-guided fine needle aspiration. 


\section{Competing interests}

The authors declare that they have no competing interests.

\section{Authors' contributions}

CDC participated in the conception and design of the study, management of samples and acquisition of data, drafting of the manuscript, and final approval of the version to be published. RB participated in the acquisition of echographic data, and critical revision of the version to be published. JCN and PHS were involved in the conception and design of the study, or acquisition of data, or analysis and interpretation of data, and critical revision of the version to be published. All authors have read and approved the final manuscript.

\section{Acknowledgements}

None to report.

\section{Received: 1 September 2015 Accepted: 22 January 2016}

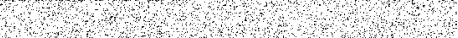

\section{References}

1. Belgian Cancer Registry. Incidence Fact Sheet. Female Breast Cancer. ICD10: C50. Belgium 2012. 2015. Accessed 1 January 2014.

2. SPF Economie, PME, Classes moyennes et Energie. Décès par groupe de causes initiales de décès, (Liste spéciale 1 de l'OMS). 2015. Accessed 1 January 2014

3. Aigner J, Schneeweiss A, Sohn C, Marmé F. The role of neoadjuvant chemotherapy in the management of primary breast cancer. Minerva Ginecol. 2011;63(3):261-74.

4. National Comprehensive Cancer Network Guidelines, version 2.2013. Accessed 1 January 2014

5. Holwitt D, Swatske ME, Gillanders WE, Monsees BS, Gao F, Aft RL, et al. Scientific Presentation Award: The combination of axillary ultrasound and ultrasound-guided biopsy is an accurate predictor of axillary stage in clinically node-negative breast cancer patients. Am J Surg. 2008;196(4):477-82.

6. Fraile M, Rull M, Julian FJ, Fuste F, Barnadas A, Llatjos M, et al. Sentinel node biopsy as a practical alternative to axillary lymph node dissection in breast cancer patients: an approach to its validity. Ann Oncol. 2000;11(6):701-5.

7. Engohan-Aloghe C, Hottat N, Noël JC. Accuracy of lymph node cell block preparation according to ultrasound features in preoperative staging of breast cancer. Diagn Cytopathol. 2010;38(1):5-8.

8. Stavros AT. Evaluation of regional lymph nodes in breast cancer patients. In: Stavros AT, editor. Breast Ultrasound. Philadelphia: Lippincott; 2004. p. 834-76.

9. Cools-Lartigue J, Sinclair A, Trabulsi N, Meguerditchian A, Mesurolle B, Fuhrer R, et al. Preoperative axillary ultrasound and fine-needle aspiration biopsy in the diagnosis of axillary metastases in patients with breast cancer: predictors of accuracy and future implications. Ann Surg Oncol. 2013;20(3):819-27.

10. Leenders MWH, Broeders M, Croese C, Richir MC, Go HLS, Langenhorst BLAM, et al. Ultrasound and fine needle aspiration cytology of axillary lymph nodes in breast cancer. To do or not to do? Breast. 2012;21(4):578-83.

11. García Fernandez A, Fraile M, Giménez N, Reñe A, Torras M, Canales L, et al. Use of axillary ultrasound, ultrasound-fine needle aspiration biopsy and magnetic resonance imaging in the preoperative triage of breast cancer patients considered for sentinel node biopsy. Ultrasound Med Biol. 2011;37(1):16-22.

12. Mills P, Sever A, Weeks J, Fish D, Jones S, Jones P. Axillary ultrasound assessment in primary breast cancer: an audit of 653 cases. Breast J. 2010;16(5):460-3.

13. Mainiero MB, Cinelli CM, Koelliker SL, Graves TA, Chung MA. Axillary ultrasound and fine-needle aspiration in the preoperative evaluation of the breast cancer patient: an algorithm based on tumor size and lymph node appearance. AJR Am J Roentgenol. 2010;195(5):1261-7.

\section{Submit your next manuscript to BioMed Central and we will help you at every step:}

- We accept pre-submission inquiries

- Our selector tool helps you to find the most relevant journal

- We provide round the clock customer support

- Convenient online submission

- Thorough peer review

- Inclusion in PubMed and all major indexing services

- Maximum visibility for your research

Submit your manuscript at www.biomedcentral.com/submit
Biomed Central 